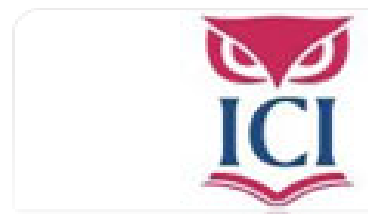

Villabella Armengol, Carlos Manuel
IUS. Revista del Instituto de Ciencias Jurídicas de Puebla A.C.

ISSN: 1870-2147

revista.ius@hotmail.com

Instituto de Ciencias Jurídicas de Puebla A. C.

México

\title{
La investigación científica en la ciencia jurídica. Sus particularidades
}

IUS. Revista del Instituto de Ciencias Jurídicas de Puebla A.C., núm. 23, 2009, pp. 5-37

Instituto de Ciencias Jurídicas de Puebla A. C.

Puebla, México 
EDITORIAL

El derecho es un producto histórico-cultural de naturaleza subjetiva, compleja, dinámica y mutable, en especial interrelación con la economía y la política, y con un marcado trasfondo filosófico y moral. En consonancia con ello, la investigación en la ciencia jurídica posee particularidades epistemológicas que tienen que ver con la connotación subjetiva de sus objetos de estudio; el carácter relativo y no demostrable empíricamente de sus esencias; la falta de exactitud cuantitativa de sus métodos; la gran sinergia de sus objetos y la variabilidad de éstos por la influencia de factores exógenos y la imposibilidad de aislarlos de la tupida red de interrelaciones en las que se encuentran.

Por estas razones, y por el predominio del enfoque empírico-positivista que ha prevalecido en la ciencia y que aún hoy no está desterrado, se ha subestimado el valor de las investigaciones sociojurídicas y no se ha privilegiado la comunicación de sus resultados.

A tenor de esto, la revista IUS en esta ocasión abandona el estilo monotemático que ha venido sosteniendo desde que inauguró su nueva época, para publicar varios resultados investigativos, muchos de ellos producto de estudios doctorales o de proyectos de investigación que realizan académicos del Instituto de Ciencias Jurídicas de Puebla y la Facultad de Derecho de la Universidad de Camagüey, instituciones entre las que existe un convenio de colaboración académica.

Encabeza los artículos, uno sobre metodología de la investigación jurídica, síntesis de una obra editada por ambos centros de educación superior.

Con lo anterior, el Instituto de Ciencias Jurídicas de Puebla muestra su voluntad por ser una institución que no sólo reproduce la ciencia jurídica en la enseñanza del pregrado y el posgrado, sino que además crea y divulga ciencia.

\section{LA INVESTIGACIÓN ,CIENTÍFICA \\ EN LA CIENCIA JURIDICA. \\ SUS PARTICULARIDADES \\ SCIENTIFIC INVESTIGATION \\ ON LEGAL SCIENCE AND ITS \\ PECULARITIES}

Carlos Manuel Villabella Armengol*

\section{RESUMEN}

El artículo, sintesis de una obra sobre metodología de la investigación en la ciencia jurídica, expone algunas de las particularidades de la investigación jurídica como parte de las ciencias sociales y establece valoraciones sobre sus nudos cognitivos. Partiendo de consideraciones generales sobre aspectos torales del diseño de investigación, como la fundamentación, el problema, la hipótesis y los métodos, realiza consideraciones sobre los matices de estos eslabones en la epistemología del derecho.

PALABRAS CLAVE: investigación juridica, ciencia jurídica, epistemología del derecho

\section{ABSTRACT}

This essay is an abstract of a work on research methodology of the Legal Science. It provides some of the peculiarities of judicial research as part of the Social Sciences and it establishes some assessments about its cognitive difficulties. Starting from general concepts on main aspects of research designing, such as the background, the problem, the hypothesis and their methods. It states some judgments about the nuances of these bonds, related to the epistemology of law.

KEY WORDS: juridical investigation, legal science, epistemology of law

* Profesor titular de la Universidad de Camagüey en Cuba y director del Programa de Doctorado en Derecho del Instituto de Ciencias Juridicas en Puebla, México. El articulo es parte de una obra titulada El proceso de investigación y comunicación en la ciencia juridica, en proceso de publicación bajo el sello editorial de la Benemérita Universidad y comunicación en la ciencia juridica, en proceso de publicación bajo
Autónoma de Puebla y el Instituto de Ciencias Jurídicas de Puebla. 


\section{SUMARIO}

1. Las ciencias sociales y la ciencia jurídica

2. Las dimensiones de la investigación en la ciencia juridica

2.1. La investigación teórica y la investigación cualitativa. Peculiaridades

3. Particularidades del proceso de investigación jurídica

3.1. La génesis de la investigación

3.2. La fundamentación de la investigación

3.3. El problema científico

3.4. La hipótesis

3.5. La estructuración de conceptos

3.6. Los métodos de investigación

\section{Las ciencias sociales y la ciencia jurídica}

Los saberes de la ciencia han sido clasificados según diferentes variables (objeto, método, concepto de ciencia, etc.) y tendencias, lo cual ha proporcionado una taxonomía no siempre coincidente y de larga data si se parte de las clasificaciones prístinas realizadas durante la Antigüedad, o incluso de la establecida por Francis Bacon y considerada como la primera desde una perspectiva moderna.

Una catalogación que nos interesa destacar es la establecida por Wilhelm Wundt a finales del siglo XIX en que distingue entre ciencias naturales y ciencias del espíritu, la cual es desplegada también por Wilhelm Dilthey (TEJEDOR, 1999: 388). Según esta noción, las ciencias naturales son aquellas cuyo objeto de estudio se encuentra en el mundo material que le es dado al hombre, en la realidad fáctica que está fuera del mismo y su objetivo es percibir esa realidad, cuantificarla y establecer las relaciones causa-efecto de los sucesos. Por su parte, las ciencias del espíritu son aquellas cuyo objeto de estudio es el mundo social creado por el hombre en el cual se desenvuelve y su objetivo es entenderlo y explicarlo.

Este enfoque dicotómico entre las ciencias dedicadas al estudio de la naturaleza y las que abordan la cultura, la sociedad, la historia y el hombre, centró durante buena parte del siglo xix la polémica respecto a si ambos conocimientos debían de considerarse como verdaderamente científicos. De esa forma, la identificación de lo social como un campo específico del saber y la aceptación de la autonomía metodológica de las ciencias que lo estudian, es el resultado de un debate nada pacífico en la epistemología en el que final- mente se rompió la hegemonía del modelo científico realista-experimental y se admitió la existencia de un esquema de métodos e instrumentos específicos para abordar los objetos de las ciencias "no naturales" y procesar los datos no empíricos que de ellas emanan.

Por ciencias sociales se considera en sentido lato al conjunto de disciplinas y áreas del conocimiento que estudian a la sociedad en sus distintas facetas, a las diferentes relaciones que en ella se entablan y al ser humano como arquitecto y protagonista de éstas; por ello han sido también reconocidas como ciencias del espíritu o ciencias de la cultura. En este horizonte se encuentra una amplia gama de áreas epistemológicas, algunas de las cuales tienen un indiscutible estatus de ciencia particular (antropología, arqueología, sociología, ciencias políticas, economía, ecología, geografía humana y social, historia, lingüística, pedagogía, etnología, demografía, derecho, psicología), mientras en otras su personalidad como disciplina autónoma es más discutible, lo cual guarda relación con el caudal de conocimientos que acumula o con perspectivas de enfoque.

Las ciencias sociales se caracterizan por lo siguientes rasgos:

1. Sus objetos de estudio son los sistemas y procesos sociales creados por el hombre y de los cuales forma parte, lo cual condiciona su naturaleza subjetiva.

2. Tienen un carácter predominantemente idiográfico, en tanto el hombre es un ser único, irrepetible y singular.

3. Los fenómenos, sistema y procesos que estudia, reciben la influencia de disimiles factores exógenos, lo cual los hace complejos, autopiéticos y sinérgicos.

4. Sus objetos de estudio son imposible de aislar de la tupida red de interrelaciones en las que se encuentran, al no ser mediante abstracción mental, por lo que necesitan ser vistos desde una perspectiva holística.

5. Parten del supuesto de que el hombre, como centro de estudio, es necesario contemplarlo como ser social, consciente, autodeterminado y mediatizado por factores sociales, psicológicos, culturales e históricos.

6. La complejidad, diversidad, inexactitud y variabilidad de sus objetos, condiciona que exista una diversidad de métodos, instrumentos, técnicas y procedimientos para investigar, a la vez que determina la imposibilidad de que éstos proporcionen una medición exacta. 
7. Las diferentes estrategias de procesamiento y análisis de la información están dirigidos fundamentalmente a la interpretación de los fenómenos que estudia.

8. Su finalidad es entender los fenómenos que estudia y comprender la realidad, por lo que es más cualitativa que cuantitativa.

9. Sus resultados siempre serán susceptibles de diversas explicaciones, no sólo por la esencia subjetiva de los fenómenos que estudia sino además por la propia subjetividad del investigador que porta prejuicios, ideologías y juicios valorativos.

10. Las fundamentaciones que establece son de escaso poder predictivo por la alta mutabilidad y relatividad de los fenómenos sociales.

11. Los sistemas explicativos que pautan tienen valor histórico-concreto, por lo que poseen un menor alcance generalizador.

En este entorno puede precisarse que el carácter de ciencia del Derecho, o dicho de otro modo, la existencia de una ciencia jurídica, es algo que ha sido cuestionado desde diferentes posturas. De un lado, están los que niegan la connotación científica no sólo del Derecho, sino también de todos los saberes sociales por el carácter subjetivo de sus objetos de estudio, la imposibilidad de trabajar con métodos que permitan demostrar con veracidad cuantitativa los resultados y la falta de exactitud de su episteme (KIRCHMANN, 1950: 51.). De otro lado, se encuentran quienes sin llegar a esta postura de raigambre empíricopositivista, sostienen que el Derecho es una ciencia periférica o pseudociencia en tanto en su contexto es muy difícil producir conocimientos realmente nuevos, en el sentido de que remueven los marcos conceptuales existentes, cambien los modelos de análisis sobre los que se basa la disciplina o renueven las estructuras metodológicas (BuENo, 1995: 88). En un tercer ángulo están los que desde el saber jurídico sostienen que el Derecho no es ciencia sino dogmática que se asume a priori, con un fin aplicativo o práctico y que no está sujeta a comprobación o demostración (KOSCHAKER 1955: 302).

Abordar las causales de estas cuestiones desborda las posibilidades de este artículo; grosso modo puede señalarse que en ello inciden factores como los siguientes: la subestimación que tuvo la investigación en el campo de las ciencias sociales hasta bien entrado el siglo xx; el predominio del paradigma científico-positivista al que correspondió la preeminencia del enfoque empírico-cuantitativo; la visión reduccionista con que se que ha enfocado el Derecho por algunas escuelas y tendencias epistemológicas que han percibido sólo la dimensión normativista del mismo, han enfatizado la faceta voluntaristaclasista que tiene, han minimizado su influencia sobre todas las estructuras societales o han sobredimensionado su función coactiva por encima de los demás roles.

El Derecho, como las demás esencias de las ciencias sociales, es un producto histórico-cultural de naturaleza subjetiva, compleja, dinámica y mutable, con especial interrelación con la económica, la política y la ideológica, y con un trasfondo filosófico y moral muy marcado; todo lo cual le proporciona una particular connotación anfibológica y multidisciplinaria.

A ello hay que agregar el carácter polisémico que intrínsecamente tiene el propio término derecho, lo cual enrarece aún más su dimensión epistemológica. Así puede señalarse que se entiende por derecho el conjunto de normas obligatorias que encauzan y organizan las relaciones sociales; el entramado de reglas que permiten el desenvolvimiento de la libertad en tanto equilibra la convivencia humana posibilitando para cada uno el máximo de libertad compatible con la libertad de los otros; el sistema de preceptos que condensan las prerrogativas o facultades subjetivas que poseen los individuos y que se legalizan en una ratio de derechos constitucionales; el sistema de cánones que pautan el marco de justicia que una sociedad acuerda para su existencia y la voluntad clasista dominante que a través de los mecanismos y procedimientos estatales se transforma en ley.

Una perspectiva más omnisciente e integral del contenido del Derecho es aquella que ha quedado contornada en el llamado enfoque tridimensional (ReAle: 1973; Aparisi, 1992: 10; AtienZA, 2001: 54; FernÁndez B. J, 2002: 4), también reconocida como teoría de los tres círculos (GARCía M., 1960: 58) o teoría integral (RecAsÉns, 1991: 88). Este enfoque sostiene que el Derecho es ante todo norma, dimensión más visible y trascendente, pero es también valor y hecho.

La dimensión normativa se encuentra determinada por ser éste un conjunto de preceptos obligatorios, imperativos, coactivos y heterónomos que conforman un sistema estructurado y coherente que tiene la finalidad de encauzar y organizar las relaciones sociales, sentido en el cual es una técnica de control y mediación que regula y coordina la coexistencia de los seres humanos, necesaria para la reproducción y continuidad de la sociedad: ubi societas, ibi ius.

La dimensión valorativa deviene de visualizar el Derecho como un sistema de cánones que pautan el marco de justicia que una sociedad acuerda para su existencia; vista ésta en un plano racional-positivo como reglas que hacen 
a los hombre iguales, que miden la conducta de éstos y que permiten dirimir conflictos entre los mismos, y en un plano emotivo como plexo axiológico que subyace en el catálogo de "hacer" o "no hacer" que postulan las normas y que se desea ponderar en las relaciones sociales.

La dimensión fáctica deviene de entender que el Derecho responde a las condiciones, problemáticas y requerimientos de una sociedad en un momento determinado, por lo que puede señalarse que en las normas lo que se hace es condensar y congelar segmentos de las relaciones humanas en los diferentes planos societales. Jus ex facto oritur plantea un viejo latinazo significando que todo derecho nace de un hecho.

Así, puede enfocarse que el Derecho es un hecho, una obra humana estimulada por la conciencia de unas necesidades en la vida social, obra producida bajo forma normativa que en su función para satisfacer esas necesidades intenta hacerlo de acuerdo con la realización de unos valores específicos (RECASÉns, 1991: 40). Es un sistema de normas que se integra en las distintas ramas del ordenamiento jurídico y que regulan ámbitos específicos de la vida en sociedad, dando lugar a un sistema que no es un simple, frío y absurdo conjunto sino que pretende alcanzar determinados valores que se vinculan a conceptos tales como la justicia, la seguridad y el bien común, los cuales son producto de una determinada cultura y de determinadas circunstancias sociales, económicas, demográficas, políticas o etnográficas (FernÁNDEz B. J., 2002: 5).

Esta perspectiva, además de que permite encuadrar de una manera multifacética y dialéctica el contenido del Derecho, posibilita a su vez hacer una decantación de tres grandes planos epistemológicos en el mismo: el normativoinstitucional, el deontológico-axiológico y el antropológico-sociológico. Ello propicia a su vez, destacar la estrecha relación de la ciencia jurídica con otras áreas del saber social como la filosofía, la sociología, la psicología, la historia, la economía y la antropología; con algunas de las cuales llega no sólo a correlacionarse sino también a compartir aristas del objeto de estudio, corporizándose así tipos específicos de saberes intermedios como la filosofía del derecho, la sociología jurídica, la historia del derecho y la antropología jurídica.

A lo anterior es conveniente agregar que el Derecho posee dos niveles gnoseológicos que es necesario tener presente para el diseño de su horizonte cognitivo, uno normativo-institucional que apuntala la existencia sistémica y coherente del ordenamiento jurídico y otro teórico-conceptual que constituye su entelequia.
El Derecho romano consolidó el término prudentia iuris o iurisprudentia, con el cual identificó el saber jurídico, visto éste fundamentalmente como actuar (agere, actio) que se desarrollaba sobre la base de un conocimiento teórico (cavere, respondere), noción que se conservó hasta que la proliferación normativa producto de la ampliación y complejización de la sociedad, el desarrollo codificador ("Codex Theodosianus", "Corpus Iuris Civiles") y el advenimiento de las primeras escuelas que enseñaban leyes, fue delineando una distinción entre el oficio de aplicar el Derecho y el quehacer de pensar el Derecho. Progresivamente esta diferenciación se ahonda hasta que en el siglo XIX termina de contornearse la noción de ciencia jurídica como disciplina académica autónoma dedicada a la sistematización, estudio y enseñanza de las normas jurídicas, independiente de la aplicación casuística-empírica de éstas. Así, se desmarca definitivamente la práctica del Derecho de la teoría del Derecho, la iurisprudentia de la scientia (MerLo, 2006).

Esta distinción, de repercusión para lo que aquí nos interesa, permite delimitar la existencia de dos niveles del saber jurídico, el teórico o científico y el práctico o dogmático. El primero se identifica con el sistema de teoremas, conceptos, principios y teorías construidos a partir de procesos lógico-racionales de sistematización y generalización, los que posibilitan el abordaje especulativo de las normas jurídicas y la enseñanza del Derecho como ciencia. El segundo se relaciona con el conjunto de técnicas que posibilitan la interpretación y realización del sistema de normas, dimensión en la cual se recompone el ordenamiento jurídico y se cierra su ciclo de vida.

Aunque bajo el rubro de dogmática jurídica se encuentran diversos enfoques en los que no es de interés profundizar en estas líneas, en general puede entenderse la misma como la tecnología que posibilita ordenar y hacer comprensible el conjunto de normas jurídicas con vistas a su aplicación; por lo que su objetivo es finalista y empírico en tanto facilita la realización del material jurídico y permite resolver el mayor número de situaciones de la forma más correcta o adecuada posible (ATIENZA, 2001: 56).

$\mathrm{Su}$ propósito por tanto no es el de especular sobre las normas, discernir sobre su creación o explicar su contenido, sino el de permitir operar eficientemente con ellas a través de una práctica hermenéutica que posibilita falsar el ordenamiento jurídico. Por ello, aunque tiene el matiz de una disciplina estructurada sobre un conjunto de conceptos y postulados, su connotación es el de un sistema de reglas y principios que viabilizan la comprensión y aplicación del Derecho. 
A tenor de lo planteado, puede señalarse que algunos de los fundamentales nudos cognitivos en donde se alojan nichos de investigación jurídica son los siguientes: el teórico-especulativo, el jurídico-filosófico, el jurídico-histórico, el jurídico-sociológico, el jurídico-comparado, el jurídico-dogmático, el jurídico-criminológico, el jurídico-politológico, el jurídico-penitenciario y el jurídico-internacional.

\section{Las dimensiones de la investigación en la ciencia jurídica}

En la literatura sobre el tema se habla de tipos de investigación para identificar las diferentes formas que éstas pueden adoptar en relación con aspectos diversos, cuestión que da lugar a una amplia y no unívoca taxonomía. Así, la tipología de la investigación guarda relación con variables como las siguientes: el formato de datos con el que se trabaje y los métodos que se requieren para interactuar con el objeto de las investigaciones (documental o de campo), el análisis de la información que realiza y el fin que se propone (cuantitativa y cualitativa), el nivel de profundidad a la que llega (exploratoria, descriptiva y explicativa), la dimensión de tiempo en la que se ubican (históricas, descriptivas-actuales, experimentales), el lapso de tiempo que abarcan (transversales $\mathrm{u}$ horizontales), el escenario en donde se desarrollen (de laboratorio, de campo o bibliográficas), la metodología que emplean (experimental, no experimental o cuasi-experimental), el propósito que tengan (pura y aplicada), etc. (ANDEREGG, 1990: 99; AchIG, 1986: 56).

Con mayor precisión es preferible hablar de dimensiones de la investigación, para concretar la delimitación del carácter teórico o empírico de la misma, la connotación cuantitativa o cualitativa y el matiz exploratorio, descriptivo, correlacional o explicativo que va a tener ésta; cuestiones que se identifican respectivamente como el tipo, el enfoque y el alcance de la investigación.

Por su tipo, la investigación científica puede ser empírica y teórica (SABINO, 1978: 146).

La investigación empírica o de campo es la que genera conocimientos a partir de la percepción que se realiza del objeto de estudio a través de las diferentes vías sensoriales, por lo que trabaja con datos factuales que se obtienen de la realidad y operan con rasgos, propiedades, manifestaciones y efectos de lo que investiga. Es un prototipo de investigación que se basa en la experiencia directa de interactuar con el objeto, cuestión por lo que es trascendente la representatividad que tenga la muestra que se va a analizar, los métodos que se emplean, la confrabilidad y validez de los mismos y los recursos para el procesamiento de la información.

Aunque por lo general toda investigación siempre se retroalimenta de teorías y paradigmas de las que parte y sobre las que estructura los nuevos conocimientos, por lo que tiene ineludiblemente un ingrediente teórico; en este tipo de investigación el quehacer empírico constituye el objeto fundamental.

La investigación teórica o documental por su parte, es la que se desarrolla sobre objetos y fenómenos que no se perciben sensorialmente, por lo cual trabaja con un dato "indirecto", especulativo y con una información abstracta que se encuentra condensada en axiomas lingüísticos diversos. Se basa en la aplicación de métodos del pensamiento lógico y genera conocimientos a partir de procesos deductivos y racionales.

La investigación empírica por su enfoque puede ser cuantitativa, cualitativa o multimodal (GRINNELL, 1997: 56).

La investigación cuantitativa está relacionada con el paradigma positivista de la ciencia y persigue como objetivo conocer el objeto de estudio a través de sus propiedades externas y manifestaciones observables, por lo cual se centra en recolectar datos del mismo, cuantificar magnitudes y hacer análisis porcentuales. Su propósito es la medición y demostración de las variables y relaciones que se han hipotetizado, para lo cual emplea un lenguaje esencialmente numérico, métodos que posibilitan la cuantificación y comprobación y muestras representativas. Tiene la finalidad de establecer patrones de comportamiento, explicar, generalizar y predecir

La investigación cualitativa se relaciona con el paradigma interpretativo y tiene como objetivo entender el objeto que estudia y evaluar las cualidades del mismo. Su propósito es destacar las relaciones y motivaciones subyacentes, por lo cual utiliza un lenguaje fundamentalmente etnográfico, métodos que permiten la descripción y penetración de lo que estudia y muestras basadas en casos-tipo. Tiene la finalidad de interpretar, comprender y destacar las cualidades de lo que estudia.

La investigación por su magnitud o alcance puede ser exploratoria, descriptiva, correlacional o explicativa (DANHKE, 1989: 385).

La investigación exploratoria o piloto por lo general inicia un camino novedoso o se efectúa sobre un objeto poco abordado, por lo cual su objetivo es sondear el tema, recopilar información preliminar, familiarizarse con la problemática, establecer prioridades o destacar facetas. Por ello, es más general 
y flexible que las demás y casi nunca constituye un fin en sí misma sino la base para subsiguientes investigaciones. En ocasiones incluso, es la base para la formulación más acabada de un problema científico.

La investigación descriptiva busca exponer las características, propiedades y manifestaciones del objeto que aborda, por lo que recolecta, ordena y jerarquiza información sobre el mismo, identifica categorías, establece relaciones, etc. Con esto brinda una comprensión general del fenómeno y esboza juicios sobre el mismo.

La investigación correlacional se centra en el análisis de la interactuación que tienen en el objeto de estudio determinadas variables o categorías, logrando aclarar la incidencia de éstas en el comportamiento del mismo y establecer regularidades y patrones de comportamiento. Logra un escalón mayor de profundidad a partir de poder brindar explicaciones sobre la base de los elementos en los que se enfoca.

La investigación explicativa por su parte es la que logra un mayor nivel de profundidad sobre el objeto ya que establece las causas de la problemática e interrelaciona desde un enfoque más general las diferentes variables, posibilitando así un entendimiento cabal del mismo y una comprensión de las regularidades y la dinámica de comportamiento del objeto que estudia.

Si nos atenemos al criterio que se ha manejado anteriormente, debe de señalarse que las investigaciones jurídicas pueden ser de tipo teórico o empírico, aunque por los objetos de estudio que se desgranan de los nudos cognitivos hay que señalar que la mayoría de las investigaciones en Derecho son de tipo teórico.

Las investigaciones jurídicas empíricas pueden ser en principio de enfoque cuantitativo o cualitativo, aunque por la textura de los objetos que se estudian son generalmente de una dimensión cualitativa.

Asimismo, éstas pueden tener un alcance exploratorio, descriptivo, corre-

lacional o explicativo, en dependencia del tema que abordan y los objetivos del investigador

Esta multilateralidad epistemológica de los objetos de la ciencia jurídica puede ilustrarse en el siguiente ejemplo: la violencia intrafamiliar, además de ser de interés para varias áreas de las ciencias sociales, puede ser abordada por el Derecho desde una dimensión teórica (la respuesta penológica a este actuar, la garantía procesal a las víctimas, la regulación de mecanismos sociales e institucionales que pueden incidir en la disminución del fenómeno, etc.), desde un enfoque empírico cuantitativo (la medición de esta problemática en un ámbito geográfico específico y la detección de las determinantes criminógenas que la provocan) y desde una metodología empírica cualitativa (la comprensión de los efectos psíquicos que tiene el fenómeno en las víctimas o los trastornos de personalidad conducentes con posterioridad a conductas delictivas). De igual forma, el mismo tópico puede conformar una investigación exploratoria (en una región en que de manera atípica se presenta un incremento de este actuar) o una explicativa (en otra en donde se desea llegar hasta ese nivel).

\subsection{La investigación teórica y la investigación cualitativa. Peculiaridades}

La investigación teórica puede definirse como aquella que trabaja con un dato ideal o especulativo contenido en objetos teórico-conceptuales, utiliza fuentes de tipo documentales primordialmente, se auxilia de métodos teóricos y emplea básicamente una semiótica gráfica para expresarse.

Este tipo de investigación puede constituir una acción investigativa en sí misma cuyo propósito es la producción de teoría científica, o una etapa imprescindible de una investigación empírica mediante la cual se relacionan los datos con un contexto teorético que permite construir los nuevos conocimientos.

Se caracteriza por los siguientes rasgos (HeRnÁNDEZ, Velia, García, 2002: 56; Martínez, 2002):

1. Los diseños de investigación que la sostienen son maleables y abiertos, en el sentido de que posibilitan cambios y ajustes en la medida en que se avanza en el proceso de investigación. En este sentido, puede señalarse que trabajan con hipótesis teóricas o ideas científicas a defender que tienen una arquitectura cognitiva más flexible.

2. Se efectúa sobre objetos teóricos cuyas magnitudes, propiedades, variables y rasgos se condensan en axiomas, teoremas, postulados, supuestos e hipótesis.

3. Su dato primario es "indirecto", intangible, especulativo, abstracto y no se percibe sensorialmente.

4. Trabaja con información que se estructura en ecuaciones gramaticales integrales, coherentes y sistematizadoras que conforman conceptos, leyes y teorías.

5. Para su ejecución interactúa con fuentes impresas o digitalizadas de disímil formato: libros, artículos, ensayos, crónicas, monografías, leyes, códigos, etcétera. 
6. No requiere de estudios pilotos ni trabajo de campo, su escenario o "campo" son la biblioteca y el centro de información.

7. No trabaja con muestras, en todo caso puede decirse que las fuentes de información que emplea tienen que ser representativas de lo que se ha escrito sobre el tema.

8. Precisa de una metodología de trabajo que posibilite la detección, exploración, selección, fichaje, análisis, extracción y compendio de las fuentes de información existentes.

9. La sustancia de los fenómenos que aborda cuya esencia es intangible, requiere de métodos que posibiliten el análisis del contenido y el desbroce de teoremas racionales.

10. El procesamiento de la información para la construcción de nuevos conocimientos precisa de mecanismos de análisis, abstracción, sintesis y generalización a un nivel lógico-racional

11. Se expresa a través de un discurso argumentativo y un lenguaje gráfico.

12. Su objetivo es construir una teoría o fundamentar, contrastar, cuestionar, refutar, reformular una que ya existe.

13. La textura de sus resultados posee un marcado sesgo ideológico en tanto una teoría es una creación ideal del ser humano, una construcción mental simbólica, derivada de determinados paradigmas teóricos.

14. Sus aportes adquieren el perfil de conceptualizaciones, teorias, revisiones críticas del sistema de conocimiento, estudios comparados, análisis desde perspectivas renovadas, reformas normativas, establecimiento de regularidades o principios, delineación de metodologías, rediseño de estructuras organizacionales o procedimientos, etcétera.

La investigación cualitativa por su parte, es aquella que persigue un fin descriptivo y omnicomprensivo del fenómeno o proceso que estudia, por lo que su epicentro es la penetración y discernimiento del objeto que aborda; el entendimiento de sus causas; el destaque de los motivos subyacentes que lo provocan; el análisis y evaluación de las variables que intervienen y la interpretación de las creencias, motivaciones e intenciones de los participantes. Para la misma, todas las perspectivas son válidas y todas las informaciones necesarias, de allí que haga visible los detalles y relevantes los pequeños significados.
Se caracteriza por los siguientes aspectos (Miles y Huberman, 1994: 27; Erlandson, 1993: 48; Strauss y CORBIN, 1990: 37-38):

1. Dirige su atención a aquellos aspectos no observables, ni susceptibles de cuantificación. Su pretensión es entender al objeto que estudia, comprender las causas y destacar las motivaciones subyacentes.

2. Puede partir de un diseño emergente y flexible, en donde el problema puede que no esté totalmente cerrado y estructurado al inicio, al igual que los objetivos no sean lo suficientemente específicos y sólo diagramen lo que se va a realizar de manera general. Asimismo, es posible que no se parta de una hipótesis acabada.

3. Trabaja con información de mayor subjetividad, se enfrenta a una mayor riqueza de datos y tiene que lidiar con variedad de matices de lo que analiza. A la par de que es capaz de manejar paradojas, incertidumbres, dilemas éticos y conflictos morales, lo cual la proyecta como un modelo humanista.

4. La relación sujeto de investigación-objeto de investigación en este modelo es intimista, inmediata, dependiente; por un lado porque el investigador se vuelve cómplice de lo que investiga y por otro porque su accionar genera influencia.

5. Igualmente, puede destacarse que el abordaje del objeto se realiza desde una perspectiva natural, estudiándolo en donde éste se encuentra, en el contexto en el que se desenvuelve; con una visión que es desde "adentro", desde el marco de referencia del mismo; de un modo que no es intrusivo a su dinámica y a través de una perspectiva holística que no lo reduce a partes aisladas o segmenta la realidad.

6. Los métodos buscan comprender y evaluar.

7. Los datos que maneja son "reales" y ricos en matices.

8. Trabaja desde un punto de vista interno del objeto y orientado al proceso.

9. Se desarrolla por lo general en pequeña escala, de manera intensiva, con muestras reducidas, a partir de casos-tipo o representativos seleccionados generalmente de manera intencional. Puede incluso que la misma no esté pre-especificada, contornándose una vez que se desarrolla el trabajo de campo.

10. No estandariza sus resultados, ni generaliza sus conclusiones; tampoco pretende establecer pautas explicativas universales.

11. Se fundamenta en procesos de análisis e inducción. 


\section{Particularidades del proceso de investigación jurídica}

\subsection{La génesis de la investigación}

En el alumbramiento de una investigación existe una fase primigenia de génesis que deviene en momento de profunda indagación y consulta, a partir de que en la misma se escudriña el entorno científico con el objetivo de decantar y perfilar el tema de investigación; lapso que resulta por lo general embarazoso para el investigador novel.

De esta forma, identificar una situación problémica, conformar una idea de investigación como punto de ignición, explorar ésta, estructurar una pregunta de trabajo que sirva de pauta y delimitar las dimensiones de la investigación, son acciones que están contenidas en esta fase de germinación de la investigación.

La culminación de esta etapa, es la escritura del diseño, proyecto o protocolo de investigación, conceptos que en muchas ocasiones se utilizan como sinónimos pero que con exactitud se refieren a ámbitos que se interconectan pero que no son equivalentes.

El diseño es la estrategia teórica-metodológica que permite abordar el objeto que se va a investigar y transformarlo, es el conjunto de operaciones que posibilitan generar nuevo conocimiento a partir del ya existente, es el núcleo que marca el metabolismo y crecimiento del conocimiento científico; aunque algunos autores emplean el término en un sentido más estrecho para referirse a la estrategia que se traza para la obtención de la información (HERNÁndEz, FERNÁNDEZ y BAPTISTA, 2003: 183).

El proyecto de investigación es un documento de licitación de la investigación que permite su aprobación y control por las instituciones que la financian o la encomiendan, por lo que contiene el diseño que sostiene su desarrollo e incluye otros aspectos metodológicos, administrativos y económicos que condicionan su realización. Es un instrumento que está en correspondencia con - el sistema de organización de la ciencia en un país y con las formalidades que estipula la entidad que convoca la investigación (SAMAJA, 1993: 202-206).

El protocolo de investigación por su parte, es una denominación que se emplea en algunos ámbitos científicos para identificar al documento que además de lo anterior, incluye cuestiones como el presupuesto de la investigación, el esquema de gastos por etapas, las fuentes de financiamiento, la identificación de los clientes o usuarios, los acuerdos o convenios de aplicación e introducción de los resultados, etcétera.
En este sentido, puede señalarse que la concepción de las investigaciones jurídicas posee algunos matices que merecen la pena destacarse.

El primero es que por lo general no existe definido dentro del sistema de la ciencia de cada país, bancos de problemas científicos en esta área del conocimiento ni están identificados temas priorizados a manera de programa que permitan encauzar o delimitar el proceso de concepción de una línea de investigación; sin embargo, ello no significa que no exista un horizonte de aristas epistemológicas sobre los que se desarrolla la actividad investigativa jurídica. Existe en realidad una diversa producción científica de diferente calibre de lo cual es expresión la gran cantidad de revistas que editan las facultades de Derecho, las instituciones jurídicas y los colegios de abogados, cuestión que constituye un medidor de la movilidad de esta ciencia.

Lo anterior sirve para pautar un segundo aspecto que particulariza la gestación y exploración de la investigación jurídica; es el arduo trabajo de indagación de la temática definida a priori y de decantación de la bibliografía suficiente y necesaria para desarrollarla, debido a la gran cantidad de fuentes existentes, tanto publicadas como digitalizadas. La orientación entre lo trascendente teóricamente y lo reiterativo o superfluo, la selección adecuada de lo que es realmente válido de todo lo que circula en internet, el no deslumbramiento por las doctrinas producidas en otros contextos geográficos, la diferencia entre lo que constituyen corrientes de pensamiento crítico y lo que es teoría decantada, son retos que hay que enfrentar en este ámbito científico y que constituyen un verdadero trance sobre todo para los investigadores jóvenes.

Un tercer elemento a comentar, que a su vez está imbricado con lo anterior, es que de la mano del desarrollo social aparecen continuamente temas novedosos que se convierten en tierra virgen para la investigación durante un tiempo (lo fue en su momento la informática, luego la temática medioambiental y más tarde el multiculturalismo acrecentado por la globalización), los que a su vez coexisten con temáticas que son inagotables por la diversidad de perspectivas que soportan (como es la teoría de la sanción penal, la institución familiar o los derechos humanos) y con materias que se renuevan porque en ellas advienen paradigmas distintos que desencadenan nuevas hipótesis (es el caso de la gobernabilidad política, el comportamiento criminógeno de un país, la supranacionalidad de instituciones jurídico-políticas que relativizan la noción de soberanía, la necesidad de una respuesta punitiva inmediata a comportamientos criminales globales, la admisibilidad del matrimonio entre 
personas de un mismo sexo, la transexualidad y la reasignación de sexo o la reproducción asistida).

\subsection{La fundamentación de la investigación}

Fundamentar una investigación significa exponer razonadamente el motivo por la cual se ejecuta la misma, cuestión que resulta trascendente a la vez que ineludible en el desarrollo de ésta.

La fundamentación como acápite estructurado que se convierte en preámbulo obligado de un proyecto, tiene la importancia de que su conformación implica que se ha indagado en la temática lo suficiente como para poder exponer de manera inteligible, coherente y resumida por qué se realiza el estudio. En ese sentido por tanto, hay que asentar que su escritura es el resultado del estudio previo de la temática mediante el cual se llega a dominar el estado del arte de ésta.

Aunque en término genérico se habla de fundamentación como un solo acto, en realidad la misma se estructura en varios tempus, ellos son: el basamento teórico del tema, la justificación de su realización, los antecedentes investigativos existentes, la novedad y la utilidad.

El basamento teórico del tema es el conjunto organizado y resumido de conceptos y datos empíricos que existen sobre el mismo, los que se plasman con el objeto de bosquejar los pilares teóricos y prácticos que tiene la investigación que se realiza. Su objetivo es establecer sobre qué conocimientos se elabora ésta.

La justificación es la necesidad de la investigación, con lo cual se deja claro la importancia que tiene el objeto de estudio, la necesidad teórica y práctica que existe para que se estudie y la trascendencia que tendrían sus resultados. Su objetivo es plasmar el por qué se hace la investigación. Ésta tiene dos momentos, uno general que contextualiza la problemática dentro de la ciencia o disciplina y dibuja el sistema problémico que alrededor de ella existe y otra particular que contextualiza el problema que se va a abordar en un entorno teórico y geográfico determinado.

Los antecedentes investigativos son las acciones investigativas que con anterioridad se han realizado sobre el tema u objeto que se estudia, lo cual es importante reflejar a modo de demostrar que el investigador las conoce y no va a incidir con su proyecto en algún aspecto ya abordado. Su objetivo es destacar qué se ha investigado y el nivel de agotamiento del tema.

La novedad está muy relacionada con lo anterior y consiste en exponer cuánto se distancia el modelo de análisis que se propone realizar de otros que se han hecho sobre el mismo objeto, cuestión por lo cual debe de condensar la contradicción conocimiento-desconocimiento que existe. Su objetivo es develar en qué se diferencia la investigación de las demás realizadas.

La utilidad consiste en distinguir el valor de los resultados que se van a obtener y que pueden concretarse en impacto teórico; beneficio práctico; efecto social; trascendencia de la metodología que emplea o aporte económico en caso de que ello pudiera ser cuantificable. Su objetivo es establecer para qué se ejecuta la investigación.

En el caso de las investigaciones jurídicas la precisión de la novedad y la utilidad del estudio es en ocasiones dificultoso, lo cual guarda relación con la textura de los aportes que se generan, la dificultad de cuantificar éstos en términos económicos y la imposibilidad de integrarlos en productos visibles.

Sería imposible esquematizar las formas en que puede haber novedad en este campo del saber, pero sí se puede precisar que ello no equivale necesariamente al descubrimiento de algo desconocido y por tanto cualitativamente nuevo. Tampoco utilidad en esta área del conocimiento es sinónimo de introducción inmediata de los resultados o comercialización de éstos.

Hay novedad en una investigación jurídica cuando se diseña o replantea una teoría, se reconceptualiza un término, se realiza un análisis desde otra perspectiva que brinda como resultado un nuevo enfoque, se ordenan estudios anteriores y con ello se madura un ángulo diferente de razonamiento o se determinan las causales de un comportamiento delictivo. Hay utilidad cuando los resultados tienen impacto teórico o trascendencia social, como en el caso de que se sistematiza una teoría, se razona la modificación de preceptos jurídicos, se fundamenta la redacción de una norma o se sugiere la introducción de nuevos procedimientos.

De esta forma, las salidas de una investigación jurídica se concretan primordialmente en resultados intangibles que inciden sobre el sistema normativo o engrosan el sistema de conocimientos que existe, conformando productos como una norma, un artículo científico o un ensayo para el uso docente e investigativo.

\subsection{El problema cientifico}

El problema científico es la expresión coherente y formalizada de una contradicción científica que se suscita ante la ausencia de conocimiento que 
imposibilita explicar un determinado fenómeno, proceso o suceso; por lo que su enunciación se constituye en el punto intermedio entre lo que se conoce y lo que se desconoce y su estructuración se convierte en el primer eslabón de la secuencia investigativa. Es una dificultad que no puede resolverse automáticamente sino que requiere de algún tipo de estudio teórico o empírico, teniendo su correcta formulación una importancia clave en tanto en su planteamiento adecuado descansa la posibilidad misma de su solución (AcHIG, 1986: 54). De este modo, puede asegurarse que la ciencia progresa no sólo por la cantidad de conocimientos que acumula sino también por la cantidad de problemas pertinentes que sea capaz de generar (Bunge, 1975: 25).

En este sentido puede enfocarse como la forma subjetiva (la problemática científica tiene que generar una preocupación en el investigador a la vez que su formulación y proyección-ejecución es producto de la experiencia y tino del mismo) en que se expresa la necesidad objetiva del desarrollo científico (deriva de problemáticas objetivas y está condicionado por el caudal científico existente).

En el proceso de formulación del problema científico, es necesario diferenciar entre una contradicción de conocimiento y la contradicción científica propiamente dicha. La primera sucede cuando la solución de la misma se logra a partir de la ordenación y sistematización del sistema de conocimientos existente, cuestión para lo cual se emplea una metódica científica pero no se elaboran nuevos conocimientos. La segunda se produce cuando los conocimientos existentes no pueden brindar respuesta adecuada a ésta (MACHADO, 2008).

La contradicción del conocimiento se supera sin necesidad de acciones investigativas, las contradicciones científicas generan problemas científicos. Para ello, tienen que ser portadoras de dos condiciones: que no existan soluciones disponibles para la misma entre los conocimientos existentes y que su solución sea posible lograrla a partir de una metodología determinada.

La contradicción científica tiene una manifestación externa y una expresión interna. La manifestación externa es observable, por lo que para su detección es suficiente que el sujeto tenga una cultura profesional y científica determinada. Ésta se formula por lo general en términos negativos, como la "ausencia" o "insuficiencia de algo"; pero ello no constituye la esencia del problema científico. La expresión interna no es observable ni se decanta a partir de la experiencia o la cultura científica y profesional, sino que necesita de una inmersión teórica y empírica del investigador en el objeto de estudio. Ésta manifiesta la esencia de la contradicción y porta la sustancia sobre la que se elabora el problema científico (Machado, 2008).
En muchas ocasiones, los investigadores noveles confunden la manifestación externa con la verdadera esencia de la contradicción y tratan de formular el problema científico sobre la misma, lo que da lugar a un problema inexacto en tanto no porta las variables reales de la contradicción científica.

En el caso de la ciencia jurídica, la concreción del problema científico atraviesa por la particularidad de que la mayoría de los objetos de estudio se encuentran en medio de una red de conocimientos que tributan a varias disciplinas o áreas del saber social, lo que le brinda carácter multidisciplinario, interdisciplinario y transdisciplinario. Por ello, constituye un aspecto importante delimitar la perspectiva científica desde la que se va abordar el objeto de estudio.

Lo anterior significa fijar el campo de acción de la investigación, es decir, delimitar aquella parte, arista o faceta del objeto que se va abordar; pero implica también concretar el ámbito científico desde el que se abordará, la perspectiva teórico-conceptual que le servirá de presupuesto y el enfoque que se le pretende dar al proceso investigativo.

Un ejemplo puede ilustrar lo que se plantea. La temática de los derechos humanos, en el sentido amplio de esta denominación, es sin lugar a dudas un objeto prioritario del Derecho como ciencia, pero lo es también de otras áreas sociales como la histórica, la economía, la sociología, la filosofía y la política. Desde un perspectiva jurídica, constituye contenido que atraviesa a numerosas disciplinas del Derecho, verbi gratia: al Derecho constitucional le interesa la legitimación que tienen en la Constitución como derechos fundamentales y sus mecanismos de garantía; el Derecho internacional se preocupa por la conformación y firma de pactos y tratados de derechos humanos regionales y universales; el Derecho civil toma una fracción de éstos, los derechos de la personalidad, como base que sostiene muchas de sus instituciones; el Derecho penal ha centrado gran parte de sus especulaciones teóricas sobre el Derecho al debido proceso, que es otro pedestal de éstos; y el Derecho laboral se desmorona como área académica ante el fenómeno globalizador y el desmontaje fáctico que se ha producido en su disciplina de los derechos laborales y sociales.

De esta manera, una investigación que tenga como contenido alguna de las aristas de los derechos humanos, tiene que encuadrarse pertinentemente desde qué disciplina del Derecho se va a abordar y qué línea discursiva va a seguir. Exempli gratia: supongamos una investigación bajo el título de "La protección jurídica del nasciturus en el ámbito extramatrimonial”. La misma, antes de llegar al meollo de lo que pretende indagar y de las tesis que desea argumentar, tiene que pronunciarse sobre una serie de nudos teóricos polémi- 
cos: el comienzo de la vida, el derecho a la vida del concebido no nacido, la titularidad y contenido de los derechos fundamentales, etc. Pero junto a esto, tiene que definir la línea científico-teórica que va a seguir, porque aunque la intención sea discurrir en el ámbito del Derecho civil, el presupuesto inicial puede partir de los derechos a la personalidad o estar más escorado al Derecho constitucional, teniendo al derecho a la vida como centro y a la anatomía de los derechos fundamentales como inflexión analítica (objeto, contenido, enunciado normativo, titularidad, regulación infraconstitucional, eficacia, limites, conflictividad con otros derechos).

\subsection{La hipótesis}

La hipótesis es un enunciado afirmativo y escueto que se plantea como conjetura o suposición fundamentada que explica y ofrece respuesta al problema científico, indicando lo que se está buscando o tratando de demostrar. Es una proposición general que se refiere a hechos no sujetos hasta ahora a la experiencia, que parte de un fundamento científico y que es corregible a la luz de los nuevos conocimientos. Por ello, puede ser llamada precisamente hipótesis, porque no es una invención sin basamento y porque se conjetura de manera provisional en espera de su verificación (Bunge, 1975: 46).

Se estructura sobre la teoría e información existente, la que funciona como presupuesto para explicar el problema, a lo cual se agrega la intuición del investigador. Toma cuerpo así una ecuación que funciona como probabilidad, presunción o predicción científica provisional que tiene que verificarse. Es así, un algoritmo sui generis del conocimiento que expresa la dialéctica conocimiento-desconocimiento, que es concebida para dar cuenta de hechos y de cuya capacidad lógica depende el nivel teórico de la investigación.

La hipótesis no debe de basarse en supuestos conceptuales insuficientemente comprobados, en prejuicios o concepciones seudocientíficas o partir de manejos estadísticos superficiales, ya que ello lastraría su capacidad de verificación científica y produciría información deformada de la realidad que investiga. Hay que tener presente que a mayor fundamentación teórica y empírica de la hipótesis, mayor solidez y contrastabilidad de la misma.

Su elaboración transcurre por un proceso en el que se parte de una suposición que es intuitiva y especulativa; luego se elabora lo que se identifica como hipótesis de trabajo con un mayor nivel de argumentación; y finalmente se elabora la hipótesis investigativa fundamentada. Ello marca una dinámica de maduración en la que ésta puede ser corregida y afinada tantas veces como sea necesario.

La hipótesis puede adquirir diversas formas en relación con el objeto de investigación, el enfoque y alcance del estudio, la cantidad de variables que plantea, la relación que establece entre las mismas, la acción de búsqueda de información que genera, etc. Sobre la tipología de las hipótesis existe una amplia y no siempre coincidente taxonomía, de la cual pueden destacarse las siguientes variantes, que son las más comunes y de mayor aplicación en las investigaciones sociales: hipótesis teórica, hipótesis descriptiva, hipótesis tipológica, hipótesis correlacional, hipótesis de grupo e hipótesis explicativa.

El imperio del paradigma positivista y el predominio del modelo de investigación empírica cuantitativa, condicionó que determinados tipos de hipótesis se perfilaran como los prototipos adecuados para establecer conjeturas de un problema científico, en tanto podían establecer mediciones certeras y explicaciones fidedignas.

De esta manera, en la ciencia jurídica es posible observar cómo en ocasiones investigadores jóvenes se esfuerzan en contornear a la fuerza hipótesis correlacionales en investigaciones que no lo necesitan por su enfoque, o se desvelan por lograr una relación variable independiente-dependiente en problemas que no lo admiten; como si de ello dependiera la validez o impacto científico de su investigación.

Por ello, no es ocioso insistir en que cada investigación de acuerdo a su tipo, enfoque y alcance requiere de un problema determinado y en consecuencia de una hipótesis específica, cuestión que es necesario subrayar en las investigaciones teóricas y empíricas cualitativas de gran presencia en la ciencia jurídica, en donde por la textura de la información con la que trabajan y el tipo de conocimiento que elaboran, muchos de los tipos de hipótesis de la investigación empírica no son aplicables.

De hecho, esta diferente complexión de la ecuación de respuesta al problema científico, sobre todo en las investigaciones teóricas, provoca que en nuestra opinión sea preferible hablar de ideas científicas en sustitución de hipótesis y de proposición o teorema en vez de variable.

\subsection{La estructuración de conceptos}

La estructuración de conceptos, definición operacional o conceptualización de variables, como se ha definido indistintamente, es el acto mediante el cual 
se definen, clarifican y hacen mensurables los conceptos y variables que se emplean en el problema e hipótesis de la investigación y que son ejes del accionar cognoscitivo. Es el acto racional mediante el cual se penetra en las propiedades y rasgos esenciales de un concepto o variable, lo cual resulta imprescindible para relacionar la investigación con la trama teórica determinada.

Tras esta denominación se solapan en realidad dos procesos de alcances diferentes. El primero, definir los conceptos que se utilizan o dimensionar el sentido en que el investigador los emplea, en caso de que sean polisémicos. El segundo, más vinculado a las investigaciones empíricas, operacionalizar las variables hasta el nivel de items o indicadores que posibiliten aplicar los métodos e instrumentos de investigación, por lo que en ese sentido definen el paso del conocimiento teórico al empírico.

En cualquier caso, este proceso supone una relación dialéctica entre el conocimiento precedente, el objeto de investigación y los propósitos del sujeto de investigación; constituyendo así la solución pragmática que el investigador ofrece al aparato conceptual y categorial de su investigación.

Los conceptos constituyen una forma ideal y abstracta de reflejar la realidad objetiva, a través de representar en una sintesis lingüística las cualidades y magnitudes que hacen a un objeto o ente identificable en sí mismo y diferenciable con otros, por lo cual puede señalarse que son las células y herramientas del conocimiento científico. Son formas de reflejo del mundo mediante las cuales se entra en conocimiento de la esencia de los fenómenos y procesos, y se generalizan los aspectos y caracteres fundamentales de los mismos (Rosental y Ludin, 1981: 75).

Un concepto o variable puede definirse de manera teórica (a partir del conocimiento que existe sobre el mismo y que se encuentra sistematizado teóricamente) o de manera práctica (a partir de lo que se percibe del mismo o se conoce a través de la experiencia sensorial), variante esta última que se constituye en la más endeble por su dimensión estrecha y no sustentable teóricamente (KeRLINGER, 2002: 75).

Los conceptos o variables pueden ser genéricos y específicos. Los primeros forman parte del caudal semántico general y aparecen definidos en diccionarios; su significado por tanto resulta tan obvio y compartido que no necesitan esclarecerse en el proyecto de investigación. Los segundos son parte del sustento teórico de una ciencia y por ende tienen una acepción específica dentro de la misma.
Pueden ser también simples y complejos. En el primer caso, éstos son definibles con facilidad por su contorno textual o porque poseen una sola dimensión. Los segundos tienen un nivel de abstracción mayor y por esto requieren de una secuencia de decantación lógico-racional hasta llevarlos al nivel de concreción que permita operar con ellos.

En las investigaciones jurídicas resulta imprescindible realizar este paso, sobre todo en el sentido de esclarecer los conceptos que se emplean, dimensionar sus facetas o delimitar el marco teórico desde el que se trabaja. Esto es preciso porque la ciencia jurídica se asienta sobre una gran cantidad de conceptos abstractos o polisémicos (Estado, gobierno, norma jurídica, derechos, democracia, gobernabilidad, relación jurídica, negocio jurídico, bien jurídico, patrimonio, dolo, culpa, etc.) o que adquieren diversas dimensiones en dependencia del paradigma del que se sustentan (iusnaturalismo, iuspositivismo, iusrealismo, idealismo jurídico, etcétera).

\subsection{Los métodos de investigación}

En sentido literal, método se deriva de las raíces griegas metá: hacia y odos: camino, por lo que su conjunción significa el camino hacia algo, el modo de proceder para lograr una meta, la vía que se sigue para llegar a un fin. Ello, aplicado al quehacer científico se traduce en el procedimiento mediante el cual se afronta un problema científico, las acciones que se aplican sobre el objeto o proceso que se estudia.

El método constituye un elemento esencial de la investigación científica en tanto posibilita el abordaje racional, argumentado, crítico y causal del objeto de estudio y condiciona el carácter científico del conocimiento que se obtiene al hacerlo demostrable y comprobable. El método científico es la manera en que la ciencia inquiere en lo desconocido, proveyendo un conjunto de prescripciones falibles y perfectibles para el planeamiento de observaciones y experimentos, para la interpretación de sus resultados y para el planteo mismo de los problemas. No se conoce otro remedio eficaz contra la fosilización y el dogma que el método científico, porque es el único procedimiento que no pretende dar resultados definitivos porque la exigencia de buscar conocimiento verificable implica un continuo inventar, probar y criticar hipótesis (Bunge, 1975: 32).

Existe una pluralidad de métodos teóricos y empíricos del conocimiento de carácter general a los que se añaden los particulares que algunos ámbi- 
tos científicos han depurado. De este arsenal, el investigador selecciona los que son más pertinentes con la naturaleza factual o racional del objeto que aborda, los objetivos que se ha propuesto, la lógica del proceso que debe de efectuar y los recursos de que dispone. No existe por tanto un recetario que funcione como camisa de fuerza para todas las investigaciones, ni la aplicación fetichista de un método determinado es lo que posibilita el éxito del proceso investigativo.

En el lenguaje coloquial se suele emplear método, instrumento y técnica de manera indistinta, aunque con exactitud no son lo mismo. El instrumento de investigación científica es la herramienta que se emplea para concretar el método, el medio a través del cual el investigador recolecta los datos y obtiene la información necesaria. Exempli gratia: la ficha de contenido que permite resumir y dominar la información existente en diferentes fuentes sobre un objeto teórico-conceptual, al que se va a aplicar el método de análisis-sintesis o la guía a partir de la cual se desarrolla la entrevista y que permite estructurar y enfocar la misma hacia los aspectos que se desean.

Las técnicas de investigación, por su parte, son las reglas y operaciones que son necesarias observar para la confección y aplicación de un instrumento de investigación a fin de que éste brinde información confiable y válida, por lo que tienen una connotación práctica y operacional. Verbi gratia: es la metódica a través de la cual se confecciona la ficha y se resume lo que está en las fuentes o las pautas para crear el rapport necesario entre el entrevistador y el entrevistado y mantener en todo momento una adecuada disponibilidad del segundo para brindar la información.

De acuerdo al objeto de investigación, los objetivos y los recursos de que se disponga, se deben de seleccionar los métodos, instrumentos y técnicas a aplicar y diseñar las acciones y pautas a seguir, conformando así una estrategia que posibilita llegar a la meta de la investigación. Esto, y no la aplicación investigativo.

Los métodos se clasifican en teóricos y empíricos.

Los métodos teóricos de investigación son procedimientos del razonamiento lógico que permiten adentrarse en las dimensiones del objeto no observables directamente, posibilitando explicar sucesos que no se encuentran a un nivel sensorial y desentrañar relaciones esenciales del mismo.

A través de estos métodos se interpretan los datos obtenidos empíricamente, se analizan y desbrozan teorías en el marco de una investigación docu-

mental y se construye el discurso científico mediante el cual se argumentan y demuestran los nuevos conocimientos.

Los métodos teóricos se presentan generalmente como acciones mentales antitéticas interconectadas dialécticamente o pares que se complementan lógicamente. Los métodos de mayor empleo son: el histórico-lógico, el de análisis-síntesis, el de abstracción-concreción, el inductivo-deductivo, el hipotético-deductivo, el sistémico-estructural-funcional, el causal, el de modelación, el genético y el dialéctico.

Los métodos empíricos son los que posibilitan captar con precisión aspectos del objeto de estudio que se encuentran a un nivel fenoménico del mismo y que son cognoscibles sensorialmente, permitiendo acumular datos e información sobre él. Son los procedimientos prácticos que propician manipular y hacer mensurable el objeto a través de sus propiedades asequibles.

No existe homogeneidad en la clasificación de éstos, además de que su diapasón se amplía si se incluyen los que postulan las ciencias particulares. Por otra parte, en ocasiones un determinado procedimiento es reconocido como método y en otras como instrumento de un método, verbi gratia: la entrevista y el cuestionario son expuestos como métodos en sí mismos (Nocedo y ABREu, 1984, v. II: 38), como instrumentos para la recopilación de información (IBARRA, 2002: 116) o como instrumentos del método de encuesta (Goode y HATT, 2002: 232). Asimismo, la observación es considerada como instrumento para la recopilación de información (RoJAs, 2001: 197) y como método empírico básico junto al experimento (ОсноА, 1997).

Los métodos empíricos de mayor empleo son: el experimento, la observación y el análisis de contenido, y asociados a éstos: la entrevista y el cuestionario como instrumentos.

Respecto a los métodos de investigación del Derecho es posible encontrar diversas posturas entre los autores que han trabajado la metodología específica de esta ciencia. Algunos retoman el caudal de métodos existente, haciendo énfasis en los teóricos como más ad hoc con el tipo de estudio no empírico que fundamentalmente se ejecuta en la ciencia jurídica (López, 2005: 56); otros mezclan métodos de investigación con tendencias y escuelas iusfilosóficas que han condicionado un modo particular de aprehender la realidad (SÁncheZ, 1998: 113); tertium genus, unos terceros se han esmerado en etiquetar métodos propios a fin de darle cuerpo claramente a una epistemología específica (SERRANO y FilHo, 1998: 87).

Consideramos que los métodos teóricos antes citados son válidos en las 
investigaciones jurídicas, con excepción quizás de la modelación y el genético. En particular son de gran aplicación la abstracción, el análisis, el examen sistémico, la inducción y el enfoque histórico.

Mediante la abstracción se aíslan los fenómenos jurídicos de su entorno e interconectividad socioeconómica, política y cultural, a la vez que pueden descomponerse desde su totalidad en los diferentes elementos o aristas que los conforman. Este proceder va acompañado irreparablemente del análisis y la síntesis, transcurso esencial en los estudios teóricos, ya que a partir del mismo se penetra el objeto que se estudia y se determinan sus propiedades, cualidades y rasgos invariantes, lo que permite conceptualizarlo y caracterizarlo. Ello es imprescindible en todas las investigaciones, pero en particular en las que abordan aristas teórico-jurídicas, iusfilosóficas, dogmáticas y politológicas.

A través de la inducción se construyen teoremas desde situaciones jurídicas particulares y casos concretos, a partir de los cuales se realizan inferencias y se establecen regularidades, obviando los atributos no determinantes y decantando lo generalizable, lo cual da pauta para establecer conclusiones e hipótesis. Ello es un proceso necesario en investigaciones que abordan normas, instituciones jurídicas o procedimientos jurisdiccionales; en estudios que tengan un matiz jurídico-sociológico o que se desarrollan sobre temas penitenciarios.

Con el método causal se pueden precisar y aislar los elementos necesarios y esenciales que provocan un fenómeno jurídico en el conglomerado pluricausal en el que están envueltos, enfocando la relación causa-efecto que se produce. Este método es decisivo en estudios criminógenos.

Utilizando un enfoque sistémico se posibilita visualizar el fenómeno dentro de la complejidad en la que se integra y delimitar su rol funcional dentro del subsistema jurídico y del sistema social del que forma parte, posibilitando asimismo desmembrar el mismo en sus diferentes estructuras y eslabones, delimitar las cualidades de éstos, precisar el conjunto de interconexiones y la escala de jerarquía de éstas, y distinguir la dinámica estructural-funcional que existe entre los mismos. Ello es un proceder valioso en investigaciones de derecho comparado, jurídico-internacionales o politológicas.

Mediante el examen histórico se analiza y desentraña la esencia de los fenómenos jurídicos, el alcance y el significado de éstos, se revela la génesis y modificación de las instituciones, se comprende la formación de los sistemas jurídicos contemporáneos, las causas de los movimientos económicos y políticos que presiden las transformaciones normativas y se caracteriza las ideas políticas y jurídicas asociadas a los mismos. Valorar desde el punto de vista histórico las normas e instituciones jurídicas o precisar la evolución y cronología de un objeto jurídico determinado es útil en todo tipo de estudio, pero es procedimiento esencial en investigaciones jurídico-comparadas, jurídico-internacionales e histórico-jurídicas.

De igual manera, los métodos empíricos reseñados, a excepción del experimento, son también de amplio empleo en la epistemología jurídica. Son utilizados sobre todo la entrevista y el cuestionario como vías complementarias de obtener información, así como el análisis de contenido para el análisis y decodificación de documentos de diversa índole.

Además de estos métodos hay que hacer mención a uno que es ponderado por algunos autores como un método específico de la ciencia jurídica, el método de derecho comparado (FIX, 1981: 91), aunque ciertamente su raíz gnoseológica, que es la comparación, es de aplicación en todos los estudios sociales.

Como etimológicamente se desprende de su enunciación, el método de derecho comparado o de comparación jurídica, es aquel mediante el cual se cotejan o contrastan dos o más objetos jurídicos (sistemas de derecho, normas, instituciones, procedimientos, etc.) a fin de descubrir sus relaciones, estimar sus diferencias y resaltar sus semejanzas, lo cual posibilita percibir los rasgos esenciales, hallar explicaciones y llegar a la esencia de las variables que se han determinado.

En este sentido, puede decirse que la tendencia contemporánea es no sólo al empleo de la comparación como recurso, sino a su vez sobre la base de ello, a la reingeniería de las normas de derecho y a la homologación de las instituciones, lo que está provocando un acercamiento interesante en los sistemas de derecho (Pizzonusso, 1987: 99).

El método de derecho comparado puede emplearse desde una doble perspectiva (Rodiere, 1965: 48; GUTTERIDGE, 1946: 1):

A. Técnico-concretizadora, cuando se estudian normas o instituciones como productos lingüísticos o técnicos.

B. Sociológica-etnográfica, cuando se enfocan como producto de unas determinadas condicionantes socio-históricas y culturales.

El estudio comparado posibilita como sistemática de estudio contornear un modelo de sabiduría normativa que racionaliza el derecho, permite perfeccio- 
nar sus normas y coadyuva a la convivencia global, a partir de convertirse en un instrumento de entendimiento que ayuda a comprender el punto de vista ajeno (ATIENZA, 1985: 291) porque un cabal conocimiento de un ordenamiento jurídico no es posible sin el conocimiento de todo lo sucedido en otra parte (Pizzorusso, 1987: 85).

Pueden señalarse también como métodos de aplicación en la ciencia jurídica, algunos que son particulares de la metodología cualitativa, como el método fenomenológico, el etnográfico y el hermenéutico.

El método fenomenológico se aplica a realidades cuya naturaleza y estructura peculiar sólo pueden ser captadas desde el marco de referencia interno del sujeto que las vive y experimenta; por tanto no puede haber una generalización en el estudio, sino que se requiere de una individualización, ya que aborda una cuestión cuya esencia depende del modo en que es vivida. Persigue como fin la descripción e interpretación de significados de vida, de esencias vividas, de aspectos existenciales del sujeto y asimismo, "ver" el mundo, la realidad, el fenómeno en estudio o al propio sujeto desde sus puntos de vista.

En este sentido, es un método de empleo en el Derecho en estudios de diagnóstico de sujetos, de estudio conductual de delincuentes, en análisis de secuelas en víctimas de delitos, en la comprensión de la estructura psíquica vivencial en reclusos, etcétera.

El método etnográfico es aquel que se aplica para estudios analítico-descriptivos de comportamientos, costumbres, creencias y prácticas sociales, por lo que es un parteaguas entre estudios de antropología cultural y de sociología cualitativa. Su objetivo es la comprensión de lo que sucede en un entorno determinado a partir de comprender el modo de vida y el comportamiento de comunidades, etnias, minorías culturales o un grupo de personas que se desenvuelve en un determinado hábitat cerrado que genera particularidades. Por ello, trata de penetrar y hacer inteligible el significado que se le brinda a determinados aspectos, las reglas de convivencia que se asumen, la estructura organizativa que se adopta, el sistema de valores y conceptos que se comparte, etc. Su meta es reconstruir las categorías específicas que los participantes usan para conceptualizar sus propias experiencias y su visión del mundo

En el caso de la ciencia jurídica es de gran utilidad en estudios que valoren el proceso de aplicación de la ley y que razonen sobre la adhesión ciudadana a la misma en un ámbito geográfico específico; en investigaciones de comprensión de factores criminógenos que dan lugar a tipologías delictivas que se presentan en una determinada comunidad a diferencia de otras; en el conocimiento y entendimiento de conductas antisociales que se producen en un grupo social concreto; en el abordaje y comprensión de aspectos que se suscitan en el ambiente carcelario; así como cuestiones relacionadas con la ejecución de la pena en estas instituciones penitenciarias.

El método hermenéutico por su parte, es aquel que posibilita entender y comprender los significados del objeto que se estudia a partir de una triple perspectiva: la del fenómeno en sí mismo, la de su engarce sistémico-estructural con una totalidad mayor, y la de su interconexión con el contexto histórico-social en el que se desenvuelve. Puede concebirse como el arte de comprensión de actos y manifestaciones humanas a partir de descifrar el contexto lingüístico y los cánones psicológicos de quien lo produce. Es el procedimiento para abordar a la realidad humana que es por esencia interpretativa.

En la ciencia jurídica este método es de un gran empleo en el estudio científico del Derecho. Sin interpretación no hay derecho, o dicho de otra manera, no hay derecho que no necesite ser interpretado, por lo que de la misma manera que un cuerpo no puede librarse de su sombra, el derecho no puede librarse de ser interpretado, estando esta actividad detrás de cada actuación de un jurista (PÉREZ RoYO, 2000: 124).

El método de investigación hermenéutico en la ciencia jurídica se matiza como:

A. Método hermenéutico-tópico: Cuando se realiza el análisis exegético de una norma jurídica, concretándose su estudio a una perspectiva técnica mediante el cual se desgrana ésta en sus diferentes estructuras hasta llegar a la célula más pequeña desde el punto de vista sintáctico. De esta manera, se aborda la norma desde la perspectiva del "discurso del objeto" o "discurso conceptista"; es decir, como documento que establece definiciones, términos y conceptos que tratan de objetivizar la realidad sobre la que se pronuncia. Se inquiere en la misma valorándola como acto lingüístico desde una perspectiva técnica-jurídica y semántica-simbólica. En este sentido, es un modelo de análisis plausible para estudios comparados en donde se cotejen productos normativos al margen de otras consideraciones.

B. Método hermenéutico-antropológico: Cuando valora la norma jurídica como un producto sociohistórico y analiza la misma como resultado cultural de una realidad histórica-concreta o examina la medida en que ésta satisfizo las expectativas factuales para la que fue creada. 
Es en este ámbito que la hermenéutica jurídica adquiere su verdadera significación.

El sentido más completo en el que se emplea el método en Derecho es en el de precisar el objetivo de la norma jurídica; valorar la correspondencia entre éste y lo que declara; hacer inteligible su estructura; develar el sistema de relaciones que establece con el resto del entramado jurídico; comprender las motivaciones teleológicas de su creación y desentrañar el condicionamiento ambiental y cultural del momento histórico que lo produjo. Para ello, es necesario tener en cuenta al menos cuatro variables: la gramatical, la teleológica, la histórica y la sistemática.

Por la variable gramatical se precisa el significado literal de un enunciado sobre la base de reglas semánticas, sintácticas y pragmáticas vigentes, determinando el significado de las palabras sobre la base de las pautas lingüísticas que gobiernan las relaciones de éstas con el enunciado y el texto marco. En este proceso no sólo cada palabra adquiere una dimensión significativa determinada en dependencia del contexto en el que se valore, sino que además cada cápsula lingüística (enunciado, fragmento, texto) adquiere también un determinado sentido.

Teniendo en cuenta la variable teleológica, se decanta la relación entre la norma objeto de estudio y su ratio, destacando la finalidad de ésta, la que puede objetivizarse en varias dimensiones: los propósitos generales de la misma, las necesidades de la disciplina jurídica o sector normativo a la que obedece, los hechos fácticos que motivaron su promulgación, etcétera.

A través de la variable axiológica se destacan los principios éticos en los que se sostiene la norma objeto de estudio, esto a partir de aceptar que el Derecho tiene una fundamentación ontológica que sustenta su andamiaje conceptual e institucional y posee un plexo axiológico que se alimenta de referentes de la nación en la que se estructura y se enraíza en la cultura histórica de la humanidad, los que son en definitiva las claves del proyecto de "deber ser" y de los presupuestos de justicia que éste propone (Villabella, 2004: 292).

Mediante la variable histórica se desentraña el entorno histórico-cultural que le dio origen, lo cual es posible a través de dos vías, haciendo inteligible las razones que motivaron al legislador en el momento de concebir la norma y el comportamiento histórico-evolutivo que ha tenido la institución en cuestión. Es por eso que puede afirmarse que detrás de toda norma existen precedentes remotos, precedentes inmediatos y circunstancias intervinientes durante los trabajos preparatorios en que se gesta.

\section{Referencias}

Achig S., Lucas, Metodología de la investigación social, Cuenca, Ecuador, Universidad de Cuenca, 1986

Ander-Egg, Ezequiel, Técnicas de investigación social, Alicante, Gráficas Díaz, 1990.

APARISI, Ángela et al., Introducción a la teoría del derecho, Valencia, Tirant lo Blanch, 1992.

AtienZA, Manuel, El sentido del derecho, Barcelona, Ariel, 2001. Introducción al derecho, Barcelona, Ariel, 1989.

BAScuÑán ValdÉs, Aníbal, Manual de técnicas de investigación jurídica, Santiago de Chile, Ediciones Jurídicas, 1961.

Bоввіо, N., Contribución a la teoría del derecho, trad. A. Ruiz Miguel, Valencia, Fernando Torres Editor, 1980.

Bueno, G., ¿Qué es la ciencia?, Oviedo, Pentalfa, 1995.

Bunge, Mario, La ciencia, su método y su filosofía, Buenos Aires, Siglo xx, 1975. La investigación cientifica, La Habana, Ciencias Sociales, 1972.

DANHKE, L. B., “Investigación y comunicación”, en Fernández-Collado, C. y DANHKE, G.

L., La comunicación humana: ciencia social, México, McGraw-Hill, 1989.

ERlandson, D. A. et al., Doing naralistic inquiri, London, Sage, 1993.

FernÁNDEZ BultÉ, Julio, Teoría del Estado y el derecho: Teoría del derecho, La Habana, Félix Varela, 2002.

Fix-Zamudio, Héctor, "Reflexiones sobre la investigación jurídica”, Revista Jurídica Messis, No. 2, 1971, pp. 34-78.

Ensayos sobre la metodología, docencia e investigaciones jurídicas, México, UNAM, 1981.

García MÁynez, Eduardo, La definición del derecho. Ensayo de perspectivismo jurídico, 2a. ed., Xalapa, Universidad Veracruzana, 1960.

GrinelL, R. M., Social work research Ct evaluation: Quantitative and qualitative approaches, 5ta. ed., Itaca, Illinois, E. E. Peacock Publishers, 1997.

Hernández Sampiere, R., Fernández-Collado, C., Baptista Lucio, Pilar, Metodología de la investigación, 3ra ed., México, McGraw-Hill, 2003.

IHERING, R. Von, ¿Es el derecho una ciencia?, Granada, Comares, 2002.

Kerlinger, F. N., LeE, H. B., Investigación del comportamiento. Métodos de investigación en ciencias sociales, México, McGraw-Hill, 2002.

Kirchmann, J. H. Von, "El carácter a-científico de la llamada ciencia del derecho", en SAvignY, F. K. Von et al. La ciencia del derecho, Buenos Aires, Losada, 1949. 
LAVAdo, Lucas, Metodología de la investigación jurídica, 2da ed., Textos Universitarios, Universidad San Martín de Porres, Lima, 2004.

López Calera, N., "Funciones del derecho”, en Garzón Valdez, E., El derecho y la justicia, 2da. ed., Madrid, Trotta, 2000.

LóPEz RuIz, Miguel, La investigación jurídica, México, unAM, 2005.

Luhmann, N., Sistema jurídico y dogmática jurídica, Madrid, Centro de Estudios Constitucionales, 1983.

MAchado RAmírez, Evelio F., "El problema científico-educativo. Estudio preliminar desde una perspectiva marxista”, Revista Pedagogía Universitaria [en línea]. Disponible en: http://revistas.mes.edu.cu/Pedagogia-Universitaria/articulos/2008/ número/189408102.pdf/view

Machado Ramírez, Evelio F., Montes de Oca Recio, Nancy, “Acerca de los llamados paradigmas de la investigación educativa: La posición teórico-metodológica fenomenológica, intuicionista, pragmática y existencialista (FIPE)”, Revista Pedagogía Universitaria [en línea]. Disponible en: http://revistas.mes.edu.cu/PedagogiaUniversitaria/articulos/2008/número/189408102.pdf/view

Martínez, M. M., "La investigación cualitativa (síntesis conceptual)", en Revista IIPSI, Vol. 9, No. 1, Facultad de Psicología, unMsm, 2006, pp. 123-126.

, Comportamiento humano. Nuevos métodos de investigación, México, Trillas, 1994.

Medawar, Peter B., Consejos a un joven científico, 2da. ed. México, Fondo de Cultura Económica, 1982.

Merlo Vega, José A., "Estilos de citas y referencias de documentos electrónicos", Revista Española de Documentación Científica, Vol. 23, No. 4, Salamanca, oct.dic., 2000, pp. 483 y ss.

Neumann, U., "La teoría de la ciencia jurídica”, en Kaufmann, A., Hassemer, W. (Coord.), El pensamiento jurídico contemporáneo, Madrid, Debate, 1992.

Nino, Carlos Santiago, Algunos modelos metodológicos de ciencia jurídica, México, Fontamara, 1993.

PÉREZ RoYo, Javier, Curso de derecho constitucional, 7 ma edición, Barcelona, Marcial Pons, 2000.

Pizzorusso, Alejandro, Curso de derecho comparado, trad. Juana Bisnozzi, Barcelona, Ariel, 1987.

Reale, Miguel, Teoría tridimensional del derecho, Santiago de Compostela, Biblioteca Hispánica de Filosofía del Derecho, 1973.

Recaséns Siches, Luis, Tratado general de filosofía del derecho, 10a. ed., México, Porrúa, 1991.

RODIERE, R., "Introducción al derecho comparado", en Revista del Instituto de Derecho Comparado, Barcelona, 1965.

Rosental, M., Ludin, P., Diccionario de filosofía, La Habana, Editora Política, 1981.

SABINo, Carlos, El proceso de la investigación, Argentina, El Cid, 1978.
SAMAJA, J., Epistemología y metodología. Elementos para una teoría de la investigación científica, Argentina, Eudeba, 1993.

SÁnchez VÁzquez, Rafael, Metodología de la ciencia del derecho, México, Porrúa, 1998.

SARLo, Óscar, "Investigación en el derecho, una cuestión problemática", Revista de la Facultad de Derecho, No. 5, Montevideo, 1992, pp. 33 y ss.

Serrano Jiménez, Pablo, Filho Heitor, Pinto, Metodología para las investigaciones jurídicas, Sao Paulo, 1998.

SuÇarez Llanos, Leonor, El derecho comparado, PORTALDERECHO [en línea], Universidad de Oviedo [9 de febrero de 2006], Disponible en: gopher://ulima.edu. pe:70/00/ccpp/seccion1/cextr/europa/granbret.txt

TEJEDOR, C. C., Historia de la filosofía en su marco cultural, Madrid, Ediciones SM, 1999.

Villabella Armengol, Carlos, "La axiología jurídica y el plexo de valores en la Constitución cubana”, Revista Jurídica, Año 4, No. 8, Cuba, julio-diciembre, 2003.

WiTKER, Jorge, La investigación jurídica, México DF, McGraw-Hill, 1995. 